\title{
Potentialities of Gene Therapy in Pediatric Endocrinology
}

\author{
Giulio Frontino $^{\text {a, b }}$ Marianna Rita Stancampiano ${ }^{\text {a }}$ Alessandro Aiuti $^{c, d, ~ e ~}$ \\ ${ }^{a}$ Department of Pediatrics, IRCCS San Raffaele Scientific Institute, Milan, Italy; ${ }^{\text {bD }}$ iabetes Research Institute, \\ IRCCS San Raffaele Scientific Institute, Milan, Italy; ' San Raffaele Telethon Institute for Gene Therapy (SR-Tiget),

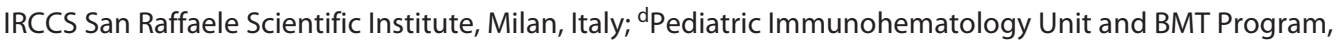 \\ IRCCS San Raffaele Scientific Institute, Milan, Italy; 'Department of Pediatrics, Pediatric Immunohematology Unit, \\ Vita-Salute San Raffaele University, Milan, Italy
}

\section{Keywords}

Gene therapy · Pediatric endocrine disease · Type 1

diabetes · Monogenic disease

\section{Abstract}

Gene therapy has become an appealing therapeutic option in many pediatric fields, including endocrinology. Unlike traditional drugs based on molecules that require repeated and frequent burdensome administrations, a single genetic therapeutic intervention may allow durable and curative clinical benefits. Although this highly innovative technology holds a great promise for the treatment of monogenic diseases, its clinical applications in the field of endocrinology have been so far challenging. In this review, we will discuss various ex vivo and in vivo approaches and potential applications of gene addition and gene editing approaches for treating hyperfunctional and hypofunctional endocrine diseases due to intrinsic defects or autoimmune origin. We will focus on the recent advances in gene therapy approaches aimed at treating type 1 diabetes and monogenic forms of endocrinopathies such as growth hormone deficiency, congenital adrenal hyperplasia, diabetes insipidus, IPEX, as well as their trends and future directions.

(c) 2021 S. Karger AG, Basel

\section{Introduction}

Thirty years since the beginning of the first clinical trials, gene therapy has become an appealing therapeutic option in many pediatric fields, including endocrinology, since a single treatment could allow obtaining lasting and curative clinical benefits, unlike traditional drugs based on molecules that require repeated administration [1-3]. Gene therapy approaches for monogenic disorders are based on the introduction of healthy copies of the gene or the precise genetic modification of the defective genes, ultimately leading to the correction of the phenotype of the disease. Gene therapy also finds its applications in various acquired conditions such as tumors infections or autoimmune diseases, with the aim of providing the modified cells with a new function with a preventive or therapeutic action, such as in the case of CAR T cells for the treatment of tumors.

The choice of the vector depends also on the target cell since an integrating vector, such as an HIV-1-derived len-

Giulio Frontino and Marianna Rita Stancampiano contributed equally to the work.
Correspondence to:

Alessandro Aiuti, aiuti.alessandro@ hsr.it 
tiviral vector, is required for replicating cells to avoid progressive dilution and loss of effect, while adeno-associated viruses (AAVs) which are predominantly nonintegrated can be used for nondividing cells.

The development of various versatile tools for genetic engineering and delivery of genes has played a key role in the progress of the field. The genetic material can be transferred employing viral vectors or nonviral methods, essentially in two ways, ex vivo or in vivo. In the ex vivo approach, the transfer of the therapeutic gene into hematopoietic stem cells or $\mathrm{T}$ cells takes place during in vitro culture by integrating vectors, mainly lentiviral vectors; at the end of the culture the genetically modified cells, i.e., the medicinal product, are administered to the patient. In the in vivo approach, the gene is delivered directly to the target cells/tissue systemically or locally (e.g., intracerebrally) and the medicinal product is represented by the viral vector. For in vivo gene therapy, vectors derived from AAV are currently used, while ex vivo gene therapy is mainly focused on the use of lentiviral vectors and gene editing with precision endonuclease [4]. Ex vivo gene therapy with autologous hematopoietic stem cells has been successfully applied to various forms of primary immunodeficiencies, neurometabolic diseases, and hemoglobinopathies, achieving so far approval in Europe as a medicinal product for severe combined immunodeficiency due to adenosine deaminase deficiency, beta thalassemia, metachromatic leukodystrophy, and X-linked adrenoleukodystrophy [1]. In vivo AAVs-mediated gene therapy has been shown to be effective in the clinic for some diseases of the central nervous system, eye diseases, metabolic diseases as well as in the deficiency of coagulation factors [5]. Two medicinal products based on in vivo gene therapy are currently approved in Europe for Spinal Muscular Atrophy [6] and retinal dystrophy (RPE65 deficiency) [7], respectively.

Typically, gene therapy for monogenic diseases has been based on approaches aimed at adding a normal copy of a gene that encodes for the defective protein (gene addition) under the control of constitutively active or tissue-specific promoters [8]. Several promoters and enhancers have been explored for different cells, including those endocrine tissues involving $\beta$-cells, adrenal, thyroid, or pituitary glands, but not all elements are easily incorporated into delivery vectors.

The recent advent of gene-editing technology could offer several advantages over traditional gene addition approaches, such as: (i) precise correction of a defective gene, (ii) introduction of a new function, (iii) inactivation of a gene involved in disease pathogenesis, and (iv) inac- tivation of a regulator gene that results eventually in correction of the disease phenotype. Gene-editing systems based on clustered regularly interspaced short palindromic repeats (CRISPR) Cas-associated nucleases and other types of engineered nucleases have proven in several preclinical studies to be versatile tools to induce DNA modification, from base substitutions to large DNA deletions. Recently, CRISPR-Cas9 technology has been employed for inhibiting the BCL11A gene to induce fetal hemoglobin production in the erythroid progeny of gene-modified hematopoietic stem cells, achieving preliminary evidence of clinical efficacy both for sickle cell and beta thalassemia [9]. Although this highly innovative technology holds great promise, many safety and efficacy issues of gene editing still need to be verified long term. Based on the above achievements, gene therapy for diabetes and other pediatric endocrinopathies has been actively pursued in the past years although mainly at the preclinical stage.

In principle, thanks to the recent technological advances both hyperfunctional and hypofunctional endocrine diseases could be amenable to gene therapy [8]. However, difficulties in delivering large genes, targeting specific endocrine organs, the need for tight control in the regulation of transgene expression, as well as the toxicity and immune response to vectors administered in vivo have been significant challenges to a wider application of gene therapy requiring in-depth investigation, further improvement and mitigation measures. Here, we will discuss the recent advances in gene therapy approaches aimed at treating type 1 diabetes (T1D) and monogenic forms of endocrinopathies such as growth hormone deficiency, congenital adrenal hyperplasia $(\mathrm{CAH})$, diabetes insipidus, IPEX, as well as their trends and future directions.

\section{Type 1 Diabetes Mellitus}

Type 1 diabetes mellitus (T1DM) is an autoimmune disorder characterized by T-cell-mediated self-destruction of insulin-secreting islet $\beta$ cells. Management of T1DM is challenging and still too frequently suboptimal even with the latest available technologies [10]. Given the strong genetic component of T1D development, gene therapy has emerged as one of the potential therapeutic alternatives to treat T1DM. Here, we will focus mainly on the current status of gene therapy approaches in preclinical studies involving cell or animal models and potential future perspectives. The main strategies investigated are based on preventing or delaying the onset of T1DM, correcting insulin 


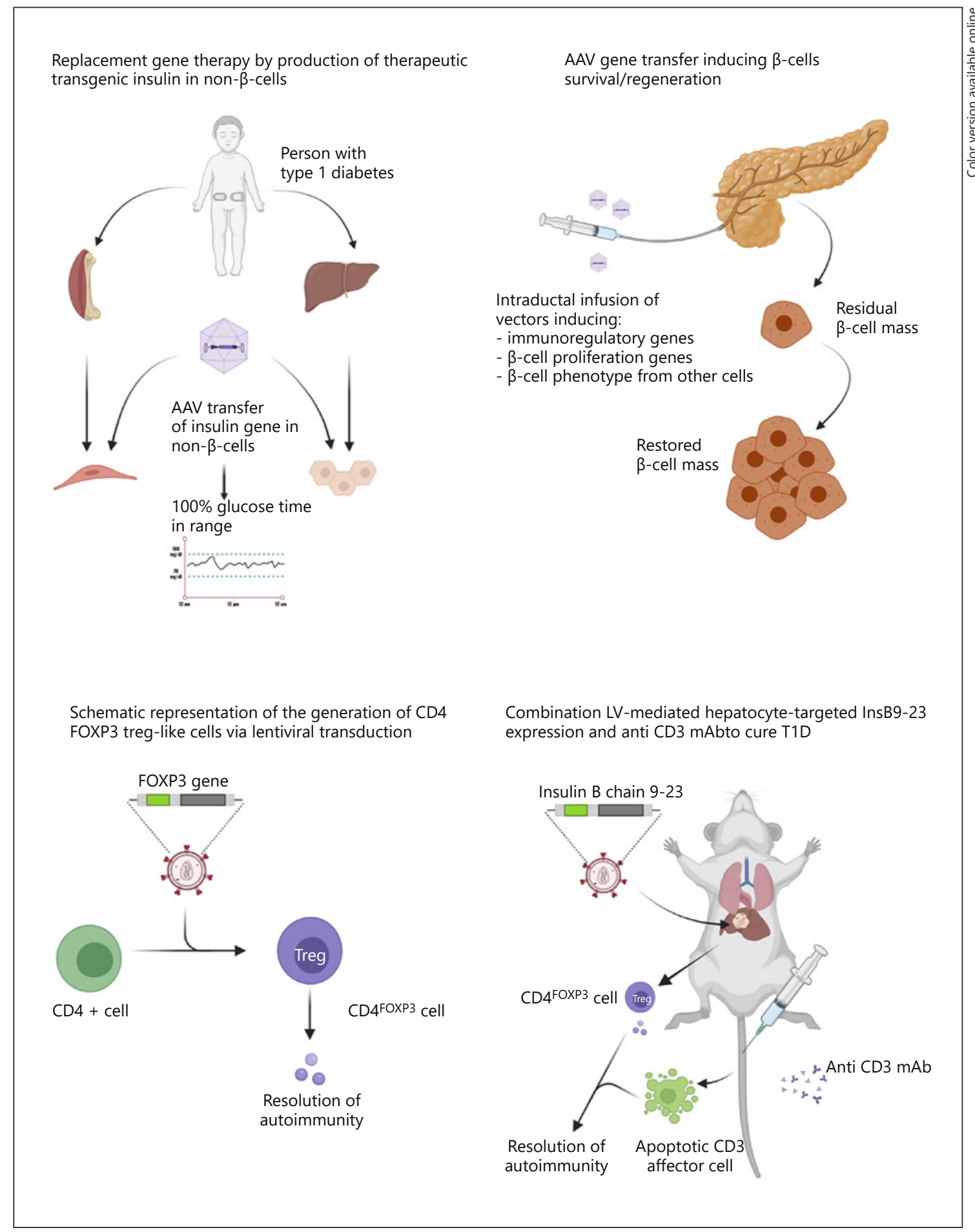

Fig. 1. Gene therapy approaches for cell and hormone replacement in T1D (created with BioRender.com).

deficiency, promoting $\beta$-cell proliferation and survival, modulating the immune/inflammatory response, and inducing insulin secretion by non- $\beta$-cells (Fig. 1 ).
Due to their presenting spontaneous autoimmunity and T1DM, nonobese diabetic (NOD) mice have been the primary animal model for studying this disease. Analo- 
gously to humans, the incidence of T1DM in NOD mice is higher in females, and they develop autoantibodies and autoreactive $\mathrm{T}$ cells prior to clinical disease onset $[11,12]$. Target $\beta$-cell antigens are also similar in both species. However, although in NOD mice insulin seems to be the eliciting antigen, in human T1D several antigens maybe instead be involved as triggers [13]. Progressive $\beta$-cell death or malfunction, autoimmune phenotypes, and progressive dysglycemia are also common in both human T1DM and NOD mice [14]. However, the early appearance of pathogenic T cells in NOD mice (as early as 5 weeks of life) followed by diffuse pancreatic insulitis by 12 weeks, reflects a possibly more aggressive nature in the animal model compared to the relatively slower onset in humans $[15,16]$. T1DM in NOD mice may not convey what triggered nor how to reverse the disease. However, this model can be sufficiently suitable to elucidate at least part of the underlying genetic and immunologic mechanisms that may aid in hampering the onset of overt hyperglycemia and identify causative gene variants that can be exploited as therapeutic pathways.

\section{$\beta$-Cell Survival and Preservation}

Induced overexpression of insulin-like growth factor 1 (IGF1) has been shown to be involved in immunomodulation and enhances $\beta$-cell survival and proliferation. Intraductal injection of an AAV encoding IGF1 has been performed in 4-week-old NOD mice to specifically transduce pancreatic cells and normoglycemia persisted in $80 \%$ of mice at 28 weeks. Furthermore, the same treatment at a time in which significant $\beta$-cell destruction is already manifest (11 weeks of age), was able to restore normoglycemia in $75 \%$ of mice [17]. Other animal studies involving induced expression of regenerating islet-derived protein 3 gamma have also shown $\beta$-cell regeneration and preservation despite autoimmune attacks [18, 19]. Furthermore, also the induced expression of glucose 6-phosphatase (G6Pase) in the liver of diabetic rat models of T1DM has been shown to induce blood glucose homeostasis. In this study, G6Pase gene expression was induced by rising glucose levels and inhibited by insulin. Interestingly, normoglycemia was achieved within a few hours of eating, and no hypoglycemia was observed [20].

Another explored candidate target gene is Klotho, an antiaging gene that is expressed in pancreatic islets in both mice and humans. Klotho deficiency is involved in $\beta$-cell apoptosis, and inducing its expression in mice under the control of a $\beta$-cell-specific promoter preserves $\beta$-cells $[21,22]$. In vitro treatment of human islet cells with the gamma-aminobutyric acid significantly in- creased Klotho expression, indicating a possible noninvasive therapeutic approach [23]. Interestingly, fibroblast growth factor 23/Klotho interaction may be substantially involved in human body metabolism and aspects of T1DM such as disease duration, insulin resistance, and development of diabetes-related complications $[24,25]$.

The $\beta$-cell mitogenic effects of ANGPTL8 (angiopoietin-like 8 ore betatrophin) are controversial. Preliminary findings suggested that overexpression of ANGPTL8 in mice models induced a 17 -fold increase in pancreatic $\beta$-cell proliferation although the direct effect of $A N G$ PTL8 as a potential target therapeutic was subsequently disputed $[26,27]$. Recent research involving a targeted gene delivery approach to deliver human ANGPTL8 gene plasmids to different organs of normal adult rats, including the pancreas, liver, and skeletal muscles compared the efficiency of $\beta$-cell replication induced by ANGPTL 8 gene in streptozotocin-induced rat models of diabetes. Improvement in glucose tolerance and fasting plasma insulin were directly associated with $\beta$-cell proliferation. Of interest, this study used ultrasound-targeted microbubble destruction as a method for organ-specific gene transfer alongside an altered insulin promoter [28].

\section{Regulated Insulin Production in Non- $\beta$-cells}

Controlled transcription and translation of proinsulin, the presence of glucose-sensing machinery, prohormone convertase expression, and a regulated secretory pathway are the key features unique to pancreatic $\beta$-cells. Gene therapy can also be exploited to induce regulated insulin production in non- $\beta$-cells. Initial studies involving genetically engineered intestinal $\mathrm{K}$ cells and hepatocytes demonstrated glucose-induced insulin secretion [29]. Recently, a single injection of an AAV encoding insulin and glucokinase genes into skeletal muscle cells of diabetic dogs was shown to be able to induce normoglycemia lasting up to 8 years [30]. Although promising, transduced cells may become susceptible to autoimmune attack alongside immune responses induced by viral vectors themselves and long-term immune tolerance still has to be demonstrated [31-33]. However, fine-tuning of viral vectors, for example, with Tet-off-inducible AAV, combined with more long-term human studies are needed before this therapeutic path may become a viable option [34].

\section{Combined Treatments}

A greater therapeutic potential may be achieved by combining gene therapy and immune modulation. Pretreatment with anti-T-cell receptor $\beta$-chain monoclonal 
antibody $(\mathrm{mAb})$ followed by hepatic gene therapy with neurogenin-3 (which determines islet lineage) and the islet growth factor betacellulin, has demonstrated sustained induction of insulin-producing cells in the liver of NOD mice, allowing for lasting reversal of new-onset or overt diabetes [35].

Targeting the T-cell receptor (TCR) with a mAb impairs T-cell response against residual and newly formed islets in overtly diabetic NOD mice. In a study by Xie et al. [35], diabetic NOD mice were transiently treated with an anti-TCR $\beta$ chain (TCR $\beta$ ) mAb, H57-597, for 5 days. Two weeks later, some NOD mice with established overt diabetes also received hepatic gene therapy using the islet-lineage determining gene Neurogenin3 (Ngn3), in combination with the islet growth factor gene betacellulin (Btc). Anti-TCR $\beta \mathrm{mAb}$ reversed more than $80 \%$ of newonset diabetes in NOD mice for $>14$ weeks by reducing the number of effector $\mathrm{T}$ cells in the pancreas. On the other hand, anti-TCR $\beta$ mAb therapy alone reversed only $20 \%$ of established overt diabetes in this model. Among those overtly diabetic NOD mice whose diabetes was resistant to anti-TCR $\beta \mathrm{mAb}$ treatment, around $60 \%$ reverted from diabetes after undergoing Ngn3-Btc hepatic gene transfer 2 weeks after initial anti-TCR $\beta$ mAb treatment. This combination of Ngn3-Btc gene therapy and antiTCR $\beta \mathrm{mAb}$ treatment induced the sustained formation of periportal insulin-producing cells in the liver of overtly diabetic mice. These data suggest that this combination therapy reverses new-onset T1D in NOD mice and protect residual and newly formed gene therapy-induced hepatic neo-islets from T-cell-mediated destruction in mice with established overt diabetes [35].

A novel approach to gene therapy for T1D involves targeting posttranscriptional modifications that give rise to pathogenic splice variants. Cytotoxic T-lymphocyteassociated antigen-4 (CTLA-4) is an immune-modulatory protein and the expression of its different splice forms has been linked to disease susceptibility or resistance in T1DM and other autoimmune diseases [36, 37]. Mourich et al. [38] employed an antisense-targeted splice switching approach to induce overexpression of the protective ligand-independent form of CTLA-4 in NOD mouse T cells in an attempt to modulate immune responses leading to T1DM CTLA-4 expressing T-cells exhibiting reduced activation, proliferation, and increased adhesion to intercellular adhesion molecule-1, similar to treatment with agonist $\alpha$-CTLA-4. Mice treated to produce a ligandindependent form of CTLA-4 at the time of elevated blood glucose levels exhibited a significant reduction in the incidence of insulitis and diabetes [38].

Gene Therapy in Pediatric Endocrine Disease
Vasoactive intestinal peptide (VIP) has shown an insulinotropic and immunomodulatory effect [39]. Because of its limited half-life due to DPP-4-mediated degradation, constant infusions or multiple injections are needed to observe any therapeutic benefit. To better exploit the therapeutic efficacy of VIP, both viral and nonviral gene delivery methods have been developed [40]. A recent study used a lentiviral vector carrying VIP gene (LentiVIP) to provide a stable VIP gene expression and test its therapeutic efficacy in multiple low-dose streptozotocininduced animal models of T1DM. LentiVIP treatment improved hyperglycemia, glucose tolerance, and prevented weight loss. Interestingly, a decrease in serum CRP levels, and serum oxidant capacity, but an increase in antioxidant capacity were also observed in treated animals. Furthermore, restoration of islet cell mass was correlated with an increase in pancreatic beta-cell proliferation. Ultimately, this strongly suggests the therapeutic effect of LentiVIP is due to immunomodulatory, insulinotropic, and cell-regenerative properties of VIP [41].

\section{Growth Hormone Deficiency}

Isolated growth hormone deficiency represents the most common pituitary hormone deficiency, and the etiology can vary from congenital or acquired causes. Causative mutations have been identified in up to $11 \%$ of cases with an isolated growth hormone deficiency, and a higher percentage of variants have been found in familial cases $(34 \%)$ and patients with severe short stature (20\%) $[42,43]$. Genetic forms of isolated growth hormone deficiency have been classified into four different groups: type IA and IB, with autosomal recessive inheritance; type II, with autosomal dominant inheritance; type III, with X-linked inheritance [44].

In these patients, conventional treatment requires daily injection of a recombinant form of growth hormone, until the final height is reached; in some cases, therapy has to be also maintained in adult age for its positive metabolic effects [45]. For these reasons, GH deficiency has been considered a possible candidate disease for gene therapy, as an alternative therapeutic strategy.

In 1991, Dhawan et al. [46] first described a systemic delivery of human growth hormone (hGH) by injection of genetically engineered myoblast into mouse muscle, using a $\gamma$-retroviral vector. After injection of transduced myoblasts, hGH could be detected in the serum for a 3 -month period. A few years later, A Al-Hendy et al. [47] reported the implantation of microencapsulated allogen- 
ic myoblasts engineered to secrete mouse growth hormone into growth hormone-deficient dwarf mice. Treated mutants showed a significant increase in linear growth, body weights, and tibial growth plate thickness than untreated controls. The allogenic myoblasts remained intact and functional for at least 6 months [47]. Implantation of subcutaneous bioartificial muscles containing skeletal myoblasts transduced with rhGH (C2-BAMs), resulted effective in attenuating muscle atrophy in mice compared with syngeneic animals receiving daily injections of rhGH [48]. In 1999, Rivera et al. [49] reported a new mechanism of regulated expression of growth hormone secretion in mice after gene transfer. They demonstrated in vivo regulation of gene expression after intramuscular injection of two separate AAV vectors, one encoding an inducible hGH target gene, and the other a bipartite rapamycinregulated transcription factor. In treated mice, basal plasma hGH expression could be induced and controlled by a rapamycin dosing regimen [49], permitting to mimic the physiological pulsatility of GH secretion [50].

Besides skeletal muscle cells, other genetically modified cell types have been used as GH delivery devices in animal models, such as fibroblasts [51, 52], bone marrow stromal cells [53], and salivary glands [54]. Recently, Higuti et al. [55] investigated a gene therapy approach based on the injection, in young and old lit/scid dwarf mice, of a plasmid in tibialis cranialis muscle encoding the hGH under the control of ubiquitin $\mathrm{C}$ promoter, followed by electrotransfer. Treatment resulted effective in promoting the catch-up growth and mIGF-I secretion, especially in young dwarf mice, which normalized IGF-I plasma levels 15 days after GT [55].

\section{Central Diabetes Insipidus}

Central diabetes insipidus (CDI) is a rare hypothalamic-pituitary disease resulting from a deficient secretion of arginine vasopressin (AVP), also known as antidiuretic hormone, from the neurohypophysis [56]. The vasopressin plays a key role in osmoregulation and water metabolism, for this reason, the symptoms characteristics of CDI are polyuria and polydipsia [57]. Genetic causes of CDI, determining a defect in AVP synthesis, represent less than $10 \%$ of cases and could be inherited as autosomal dominant, autosomal recessive, or X-linked recessive traits [57].

In contrast to $\mathrm{GH}$, there are very few reports on gene therapy as a treatment for CDI. Geddes et al. [58] reported that the stereotactical injection of an adenoviral vector encoding the arginine vasopressin cDNA (AdAVP) into the supraoptic nuclei of the hypothalamus of an AVPdeficient Brattleboro rat, resulted in a restored expression of AVP in magnocellular neurons. AVP production determined a reduced daily water intake and urine volume as well as an increased urine osmolarity, lasting for up 4 months [58]. More recently, Yoshida et al. [59] describes a different GT approach, using the skeletal muscle as a target tissue for transgene expression. They documented that the bioactive form of AVP could be produced by non-endocrine cells with a modified vasopressin gene containing a ubiquitous endoprotease furin cleavage site. Based on this approach, using the electroporation technique, the furin-processable vasopressin gene was introduced into the skeletal muscle of AVP-deficient Brattleboro rats, determining a significant reduction in urine volume and an increased urine osmolality, for approximately 3 weeks [59].

\section{Congenital Adrenal Hyperplasia}

CAH refers to a group of diseases determined by the reduced or absent activity of one of the enzymes involved in the regulation of the glucocorticoid and/or mineralocorticoid synthesis in the adrenal gland. The most common form of CAH is represented by 21-hydroxylase deficiency, an autosomal recessive disorder caused by pathogenetic variants in the CYP21A2 gene. Mutations in CYP21A2 (P450c21) determine impaired adrenocortical production of cortisol and the accumulation of the steroid precursors upstream of the defective enzyme, which are shunted into the preserved androgen pathway [60]. Patients affected by CAH require lifelong and lifesaving glucocorticoid and mineralocorticoid replacement therapy. However, since adrenal physiology is not precisely restored, affected individuals may experience adverse long-term outcomes in terms of growth, metabolic, reproductive, and mental health endpoints [61]. Moreover, patients remain at significant risk of adrenal crises [62], and recent studies have revealed an increased risk of respiratory, urinary, and gastrointestinal infections and allcause mortality compared to the general population $[63$, 64].

As a monogenic disease caused by loss-of-function mutations, $\mathrm{CAH}$ would be considered a candidate for the gene therapy approach. The first attempt of gene therapy in $21 \mathrm{OH}$-deficient mice $(21 \mathrm{OH}-)$ was published by Tajima et al. [65] using a replication-deficient adenovirus containing the genomic sequence of human CYP21 


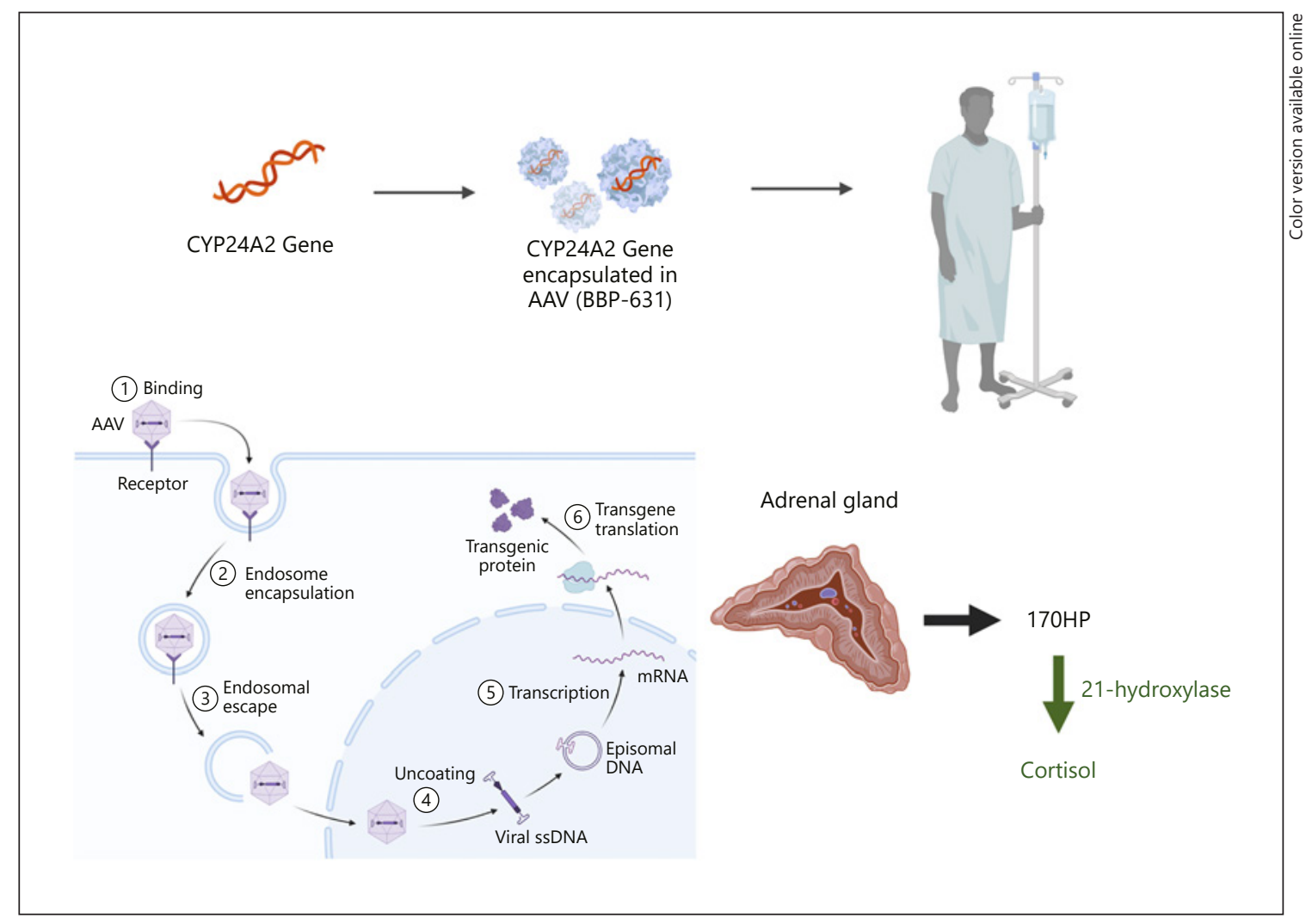

Fig. 2. Phase $1 / 2$ open-label gene therapy in adults with 21OHD-CAH through administration of an AAV5 encoding the human CYP21A2 gene (BBP-631) (NCT04783181) (created with BioRender.com). 17OHP, 17-hydroxyprogesterone.

(hAdCYP21). The intra-adrenal injection of hAdCYP21 in $21 \mathrm{OH}$ - animal models induced a hCYP2 1 mRNA expression with an increased concentration of plasma corticosterone, from undetectable levels to levels similar in wild-type mice, lasting up to 40 days [65]. More recently, an AAV gene therapy strategy was developed based on the intravenous injection of an AAVrh10-CAG-humanCYP21A2-HA vector in Cyp21 $1^{-/}$mice models. Treated mice showed an increased body weight and near normalization of urinary progesterone for more than 15 weeks, improved response to stress, and restoration of near-normal expression of several important genes in the adrenal cortex [66]. In 2020, Eclov et al. [67] reported the attempt to treat cynomolgus monkeys with increasing doses of a nonreplicating $\mathrm{rAAV} 5$ vector containing ssDNA of the human CYP21A2 transgene (BBP-631). In treated animal models, vector genome copies and hCYP21A2 RNA expression were present in the liver and adrenals at 4 and 24 weeks, in a dose-dependent manner; no adverse events were documented [67]. On this path, in 2021, Merke et al. [68] presented the design of a phase $1 / 2$ open-label, dose-escalation study of the safety and efficacy of gene therapy in adults with 21OHD-CAH through administration of an AAV5 encoding the human $C Y$ P21A2 gene (BBP-631) (NCT04783181) which is currently enrolling patients (Fig. 2). Primary endpoints will be safety evaluation and selection of the optimal drug dose, while secondary outcomes include changes in 17-OHP, endogenous cortisol, and androstenedione. This first clinical study could represent a milestone in the development of new strategies for the treatment of patients affected by CAH.

\section{Monogenic Autoimmune Endocrinopathies}

Autoimmune disorders affect often endocrine glands as single or multiple targets, resulting in hormone hyperproduction or hypoproduction. Monogenic autoimmune disorders include inborn errors of the immune system which alter central and/or peripheral tolerance to autoantigens such as IPEX, AIRE, ALPS, or deficiency of RAG1, 
CTLA4, LRBA, or gain of function mutations in genes such as PIK3CD (activated phosphoinositide 3-kinase $\delta$ syndrome (APDS), STAT1, or STAT3) [69]. Chronic immune suppression can be effective in controlling clinical manifestations but carrying the serious burden of side effects, including the risk of infections and malignancies. The improved ability to define the molecular defect and pathophysiology of these disorders has resulted in the development of targeted therapeutic interventions with biological drugs on $\mathrm{mAbs}$ or drugs acting on specific pathways. Gene therapy approaches have been aimed at correcting the genetic defect in hematopoietic stem cells and/ or lymphocytes by gene addition or gene editing.

IPEX is a paradigmatic example of a rare, often fatal, $\mathrm{X}$-linked monogenic immune dysregulation disorder that typically presents during infancy with a triad of enteropathy, autoimmune endocrinopathy, and dermatitis. IPEX is caused by mutations in the gene FOXP3, which encodes a transcription factor necessary for the maintenance of immunologic tolerance by thymus-derived regulatory T (Treg) cells [70]. Hematopoietic stem-cell transplantation (HSCT) is the only curative therapy available to IPEX patients and it is offered to those with low organ involvement pretransplant, as it promises definitive therapy with disease resolution and better quality of life as compared with chronic immune suppressive therapy. Many of the features of IPEX remit after successful transplantation, although endocrinopathies frequently persist due to permanent organ damage. In the review of 96 patients mentioned above, 58 underwent HSCT, with a 15 -year overall survival rate of $73.2 \%$ [71]. However, potential complications of HSCT in IPEX patients include macrophage activation syndrome, infection, graftversus-host disease, and growth failure. Alternative approaches to HSCT are being pursued, such as gene therapy or gene editing of autologous hematopoietic stem cells or lymphocytes. In a first approach, $\mathrm{CD} 4^{+} \mathrm{T}$ cells from healthy donors and IPEX were converted into functional Treg-like cells by lentiviral transfer of FOXP3 [72]. These engineered Treg-like cells in humanized mice models protected from GvHD and hyper-proliferation of $\mathrm{CD} 4^{+}$memory T cells. At the same time, these engineered Treg-like cells maintain in vivo expansion of antigenprimed $\mathrm{T}$ cells or tumor clearance in the mice model. These data support the clinical application for IPEX syndrome and other immune-mediated diseases.

An alternative approach is based on gene editing of FOXP3. Recent studies in vitro and in vivo animal models have shown successful CRISPR-based gene correction with the appropriate expression of FOXP3 protein in ed- ited Tregs derived from IPEX patients [73]. Although technically in its infancy as a therapeutic tool, these investigational data suggest the feasibility of gene editing.

\section{Conclusions and Perspectives}

Gene therapy for endocrinological pediatric disorders is an area of increasing interest although still challenging. Several approaches for diabetes type I have been pursued at the preclinical level but have not yet reached the clinical stage. Gene therapy for the cerebral form of X-linked adrenoleukodystrophy has been recently approved in the European Union, but similarly to allogeneic HSCT, the treatment is not acting on the adrenal insufficiency. The first clinical trial based on in vivo delivery of the therapeutic with AAV vectors for $\mathrm{CAH}$ has recently started and will provide important information on the safety and efficacy of this strategy.

In principle, monogenic diseases causing endocrine hypofunction could be amenable through the delivery of the wild-type gene coding for the missing/defective hormone or gene editing by homology-directed repair. On the other hand, strategies for amending endocrine hyperfunction may require either the transfer of a gene with ameliorating effects or the inhibition of genes associated with hormone hypersecretion by the use of antisense sequences, miRNA, or deletion/inactivation by gene editing through nonhomologous end-joining.

The remarkable scientific and technological advances in gene modification and delivery offer a unique scenario for the future for both hyperfunctional and hypofunctional endocrine diseases. Improved high-fidelity nucleases, base editing, and prime editing represent promising technologies for efficient and regulated engineering [74, 75]. Epigenomic engineering through CRISPR-based editing or other approaches aimed at targeting chromatin and regulating genes represents another promising tool for controlling biological functions and harnessing altered gene expression [76].

It should be considered that gene-editing technology is relatively new and there is still limited clinical experience on the immunogenicity of their components, the safety of off-target insertions, and of large deletions and rearrangements that occur close to the recombination site. In addition, its delivery still requires classical viral vectors such as AAV and lentiviral vectors. As an alternative, nonviral gene delivery vehicles such as nanoparticles have also been developed to mitigate the side effects associated with viral delivery carriers [77]. They have dem- 
onstrated very good biocompatibility/biodegradability, low immunogenicity, and easily adjustable properties. Nanotechnological advancements allow delivery of nucleic acids, potentially enhancing the performance of precision medicine therapies, thus accelerating their clinical translation. An example of the power of this new technology derives from preliminary data of an in vivo gene-editing therapy for transthyretin amyloidosis, a rare multisystem disease caused by a protein synthetized in the liver that misfolds and aggregates in selected tissues. A single dose of lipid nanoparticle encapsulating messenger RNA for Cas9 protein and a single guide RNA targeting the disease-causing gene showed an initial favorable safety profile along with early evidence of efficacy in preclinical models and patients [78].

For endocrine diseases caused by acquired and genetic autoimmune diseases, therapeutic approaches exploiting Tregs are of great interest since these cells play a crucial role in maintaining tolerance to self-antigens and nonharmful foreign antigens. Genetically engineered $\mathrm{T}$ cells to express normal FOXP3 have been proposed to treat IPEX syndrome and other immune-mediated diseases caused by insufficient or dysfunctional FOXP3 ${ }^{+}$Tregs. In addition, Treg immunotherapies using ex vivo isolated or in vitro expanded Tregs have been tested in clinical trials for several diseases, including graft-versus-host disease and early-onset T1D [72, 79, 80]. Another interesting strategy suitable for endocrinopathies and tested in preclinical studies is a combined approach of antigen-specific immunotherapy based on antibody-mediated therapy (anti-CD3) and gene transfer into the liver of insulin $\beta$-chain peptide, which cooperate to control diabetogenic cells [81].

The safety of these approaches should also be considered and well balanced with the expected benefit and available treatments. Hematopoietic stem cell gene therapy with gamma-retroviral vectors has been complicated with the onset of myelodysplastic syndromes and leukemias, due to the integration of the provirus in some proto-oncogenes such as MECOM and LMO2 $[82,83]$. Lentiviral vectors have shown in the past decade an excellent safety track record although recently, one participant in a clinical trial for the treatment of adrenoleukodystrophy developed a myelodysplastic syndrome [84].

In clinical trials using rAAV vectors, various adverse events have been reported, such as hepatotoxicity, thrombotic microangiopathy, and dorsal root ganglia toxicity [85]. Moreover, many aspects regarding the interaction of AAV vectors and the human host, such as vector immunogenicity, therapeutic potency, persistence, and po- tential genotoxicity due to integrations in the human genome, have to be further elucidated [86].

In summary, there is a wide armamentarium of gene therapy approaches for endocrinopathies due to endocrine gene defects or autoimmune disorders offering the potential of a single therapeutic intervention with durable benefit. Some of these approaches have reached the preclinical proof of concept stage and are now moving to clinical applications to investigate their safety and efficacy.

\section{Acknowledgments}

We thank Fondazione Telethon for support. A.A. is the recipient of the Else Kröner Fresenius Prize for Medical Research 2020. A.A. is a member of the European Reference Network (ERN) for Rare Immunodeficiency, Autoinflammatory and Autoimmune Diseases (Project ID No 739543).

\section{Conflict of Interest Statement}

A.A. is the PI of clinical trials sponsored by Orchard Therapeutics. The other authors have nothing to declare.

\section{Funding Sources}

The funders had no role in preparation of the review.

\section{Author Contributions}

G.F., M.R.S., and A.A. participated in drafting the review. A.A. revised it critically for the intellectual content and gave final approval of the version to be submitted.

References

1 Ferrari G, Thrasher AJ, Aiuti A. Gene therapy using haematopoietic stem and progenitor cells. Nat Rev Genet. 2021;22(4):216-34.

2 Tucci F, Scaramuzza S, Aiuti A, Mortellaro A. Update on clinical ex vivo hematopoietic stem cell gene therapy for inherited monogenic diseases. Mol Ther. 2021;29(2):489-504.

3 High KA, Roncarolo MG. Gene therapy. N Engl J Med. 2019 Jul 31;381(5):455-64.

4 Staal FJT, Aiuti A, Cavazzana M. Autologous stem-cell-based gene therapy for inherited disorders: state of the art and perspectives. Front Pediatr. 2019 Oct 31;7:443.

5 Mendell JR, Al-Zaidy SA, Rodino-Klapac LR, Goodspeed K, Gray SJ, Kay CN, et al. Current clinical applications of in vivo gene therapy with AAVs. Mol Ther. 2021 Feb 3;29(2):46488. 
6 Kirschner J, Butoianu N, Goemans N, Haberlova J, Kostera-Pruszczyk A, Mercuri E, et al. European ad-hoc consensus statement on gene replacement therapy for spinal muscular atrophy. Eur J Paediatr Neurol. 2020 Sep;28:38-43.

7 Wang X, Yu C, Tzekov RT, Zhu Y, Li W. The effect of human gene therapy for RPE65-associated Leber's congenital amaurosis on visual function: a systematic review and metaanalysis. Orphanet J Rare Dis. 2020;15(1):49.

8 Barzon L, Bonaguro R, Palù G, Boscaro M. New perspectives for gene therapy in endocrinology. Eur J Endocrinol. 2000;143(4): $447-66$.

9 Frangoul H, Altshuler D, Cappellini MD, Chen YS, Domm J, Eustace BK, et al. CRISPR-Cas9 gene editing for sickle cell disease and $\beta$-thalassemia. N Engl J Med. 2020 Dec $5 ; 384(3): 252-60$.

10 Hernando ME, García-Sáez G, Gómez EJ, Pérez-Gandía C, Rodríguez-Herrero A. Automated insulin delivery: the artificial pancreas technical challenges. Am J Ther. 2020 Jan/Feb27(1):e62-70.

11 Melanitou E, Devendra D, Liu E, Miao D, Eisenbarth GS. Early and quantal (by litter) expression of insulin autoantibodies in the nonobese diabetic mice predict early diabetes onset. J Immunol. 2004 Dec;173(11): 6603-10.

12 You S, Belgith M, Cobbold S, Alyanakian MA, Gouarin C, Barriot S, et al. Autoimmune diabetes onset results from qualitative rather than quantitative age-dependent changes in pathogenic T-cells. Diabetes. 2005 May;54(5):1415-22.

13 Atkinson MA. The pathogenesis and natural history of type 1 diabetes. Cold Spring Harb Perspect Med. 2012 Nov 1;2(11):a007641.

14 DiLorenzo TP, Graser RT, Ono T, Christianson GJ, Chapman HD, Roopenian DC, et al. Major histocompatibility complex class I-restricted $T$ cells are required for all but the end stages of diabetes development in nonobese diabetic mice and use a prevalent $\mathrm{T}$ cell receptor alpha chain gene rearrangement. Proc Natl Acad Sci U S A. 1998 Oct;95(21):1253843.

15 Leete P, Willcox A, Krogvold L, Dahl-Jørgensen K, Foulis AK, Richardson SJ, et al. Differential insulitic profiles determine the extent of $\beta$-cell destruction and the age at onset of type 1 diabetes. Diabetes. 2016 May 1; 65(5):1362-9.

16 Campbell-Thompson M, Fu A, Kaddis JS, Wasserfall C, Schatz DA, Pugliese A, et al. Insulitis and $\beta$-cell mass in the naturalh history of type 1 diabetes. Diabetes. 2016 Mar 1; 65(3):719-31.

17 Mallol C, Casana E, Jimenez V, Casellas A, Haurigot V, Jambrina C, et al. AAV-mediated pancreatic overexpression of Igf1 counteracts progression to autoimmune diabetes in mice. Mol Metab. 2017 Jul;6(7):664-80.
18 Parikh A, Stephan AF, Tzanakakis ES. Regenerating proteins and their expression, regulation and signaling. Biomol Concepts. 2012 Feb;3(1):57-70.

19 Li S, Huang L. Nonviral gene therapy: promises and challenges. Gene Ther. 2000 Jan; 7(1):31-4.

20 Chen R, Meseck ML, Woo SL. Auto-regulated hepatic insulin gene expression in type 1 diabetic rats. Mol Ther. 2001 Apr;3(4):58490.

21 Lim K, Groen A, Molostvov G, Lu T, Lilley KS, Snead D, et al. a-Klotho expression in human tissues. J Clin Endocrinol Metab. 2015 Oct;100(10):E1308-18.

22 Lin Y, Sun Z. Antiaging gene klotho attenuates pancreatic $\beta$-cell apoptosis in type $1 \mathrm{dia}-$ betes. Diabetes. 2015 Dec;64(12):4298-311.

23 Prud'homme GJ, Glinka Y, Kurt M, Liu W, Wang $\mathrm{Q}$. The anti-aging protein klotho is induced by GABA therapy and exerts protective and stimulatory effects on pancreatic beta cells. Biochem Biophys Res Commun. 2017 Dec;493(4):1542-7.

24 Berezin AE, Berezin AA. Impaired function of fibroblast growth factor 23/klotho protein axis in prediabetes and diabetes mellitus: promising predictor of cardiovascular risk. Diabetes Metab Syndr. 2019 Jul;13(4):254956.

25 Flotyńska J, Uruska A, Araszkiewicz A, Zozulińska-Ziółkiewicz D. Klotho protein function among patients with type 1 diabetes. Endokrynol Pol. 2018 Dec;69(6):696704.

26 Cox AR, Barrandon O, Cai EP, Rios JS, Chavez J, Bonnyman CW, et al. Resolving discrepant findings on ANGPTL8 in $\beta$-cell proliferation: a collaborative approach to resolving the betatrophin controversy. PLoS One. 2016 Jul;11(7):e0159276.

27 Yi P, Park JS, Melton DA. Retraction notice to betatrophin: a hormone that controls pancreatic $\beta$ cell proliferation. Cell. 2017 Jan; 168(1):326.

28 Chen S, Shimoda M, Wang MY, Ding J, Noguchi $\mathrm{H}$, Matsumoto S, et al. Regeneration of pancreatic islets in vivo by ultrasound-targeted gene therapy. Gene Ther. 2010 May; 17(11):1411-20.

29 Cheung AT, Dayanandan B, Lewis JT, Korbutt GS, Rajotte RV, Bryer-Ash M, et al. Glucose-dependent insulin release from genetically engineered K cells. Science. 2000 Dec; 290(5498):1959-62.

30 Jaén ML, Vilà L, Elias I, Jimenez V, Rodó J, Maggioni L, et al. Long-term efficacy and safety of insulin and glucokinase gene therapy for diabetes: 8-year follow-up in dogs. Mol Ther Methods Clin Dev. 2017 Sep;6:17.

31 Touchefeu Y, Harrington KJ, Galmiche JP, Vassaux G. Review article: gene therapy, recent developments and future prospects in gastrointestinal oncology. Aliment Pharmacol Ther. 2010 Oct;32(8):953-68.
32 Ramshur EB, Rull TR, Wice BM. Novel insulin/GIP co-producing cell lines provide unexpected insights into Gut K-cell function in vivo. J Cell Physiol. 2002 Sep;192(3): 339-50.

33 Ren B, O'Brien BA, Swan MA, Koina ME, Nassif N, Wei MQ, et al. Long-term correction of diabetes in rats after lentiviral hepatic insulin gene therapy. Diabetologia. 2007; 50(9): 1910-20.

34 Gan SU, Fu Z, Sia KC, Kon OL, Calne R, Lee KO. Development of a liver-specific tet-off AAV8 vector for improved safety of insulin gene therapy for diabetes. J Gene Med. 2019 Jan;21(1):e3067.

35 Xie A, Li R, Jiang T, Yan H, Zhang H, Yang $\mathrm{Y}$, et al. Anti-TCR $\beta \mathrm{mAb}$ in combination with neurogenin 3 gene therapy reverses established overt type 1 diabetes in female NOD mice. Endocrinology. 2017 Oct; 158(10):3140-51.

36 Ueda H, Howson JM, Esposito L, Heward J, Snook H, Chamberlain G, et al. Association of the T-cell regulatory gene CTLA4 with susceptibility to autoimmune disease. $\mathrm{Na}$ ture. 2003 Apr;423(6939):506-11.

37 Chen Y, Chen S, Gu Y, Feng Y, Shi Y, Fu Q, et al. CTLA- $4+49 \mathrm{G} / \mathrm{A}$, a functional T1D risk SNP, affects CTLA-4 level in Treg subsets and IA-2A positivity, but not beta-cell function. Sci Rep. 2018 Jul;8(1):10074.

38 Mourich DV, Oda SK, Schnell FJ, Crumley SL, Hauck LL, Moentenich CA, et al. Alternative splice forms of CTLA- 4 induced by antisense mediated splice-switching influences autoimmune diabetes susceptibility in NOD mice. Nucleic Acid Ther. 2014 Mar; 24(2):114-26.

39 Ganea D, Hooper KM, Kong W. The neuropeptide vasoactive intestinal peptide: direct effects on immune cells and involvement in inflammatory and autoimmune diseases. Acta Physiol. 2015 Feb;213(2):442-52.

40 Herrera JL, Fernández-Montesinos R, González-Rey E, Delgado M, Pozo D. Protective role for plasmid DNA-mediated VIP gene transfer in non-obese diabetic mice. Ann N Y Acad Sci. 2006;1070:337-41.

41 Erendor F, Sahin EO, Sanlioglu AD, Balci MK, Griffith TS, Sanlioglu S. Lentiviral gene therapy vectors encoding VIP suppressed diabetes-related inflammation and augmented pancreatic beta-cell proliferation. Gene Ther. 2021;28(3-4):130-41.

42 Mullis PE. Genetic control of growth. Eur J Endocrinol. 2005;152(1):11-31.

43 Alatzoglou KS, Dattani MT. Genetic causes and treatment of isolated growth hormone deficiency-an update. Nat Rev Endocrinol. 2010;6(10):562-76.

44 Procter AM, Phillips JA III, Cooper DN. The molecular genetics of growth hormone deficiency. Hum Genet. 1998;103(3):255-72. 
45 Grimberg A, DiVall SA, Polychronakos C, Allen DB, Cohen LE, Quintos JB, et al. Guidelines for growth hormone and insulinlike growth factor-I treatment in children and adolescents: growth hormone deficien$c y$, idiopathic short stature, and primary insulin-like growth factor-I deficiency. Horm Res Paediatr. 2016;86(6):361-97.

46 Dhawan J, Pan LC, Pavlath GK, Travis MA, Lanctot AM, Blau HM. Systemic delivery of human growth hormone by injection of genetically engineered myoblasts. Science. 1991 Dec 6;254(5037):1509-12.

47 Al-Hendy A, Hortelano G, Tannenbaum GS, Chang PL. Correction of the growth defect in dwarf mice with nonautologous microencapsulated myoblasts: an alternate approach to somatic gene therapy. Hum Gene Ther. 1995 Feb 1;6(2):165-75.

48 Vandenburgh H, Tatto MD, Shansky J, Goldstein L, Russell K, Genes N, et al. Attenuation of skeletal muscle wasting with recombinant human growth hormone secreted from a tissue-engineered bioartificial muscle. Hum Gene Ther. 1998 Nov 20;9(17): 2555-64.

49 Rivera VM, Ye X, Courage NL, Sachar J, Cerasoli F Jr, Wilson JM, et al. Long-term regulated expression of growth hormone in mice after intramuscular gene transfer. Proc Natl Acad Sci U S A. 1999 Jul 20;96(15): 8657-62.

50 Hartman ML, Iranmanesh A, Thorner MO, Veldhuis JD. Evaluation of pulsatile patterns of growth hormone release in humans: a brief review. Am J Hum Biol. 1993 Jan 1;5(6): 603-14.

51 Chen BF, Chang WC, Chen ST, Chen DS, Hwang LH. Long-term expression of the biologically active growth hormone in genetically modified fibroblasts after implantation into a hypophysectomized rat. Hum Gene Ther. 1995 Jul 1;6(7):917-26.

52 Cheng WT, Chen BC, Chiou ST, Chen CM. Use of nonautologous microencapsulated fibroblasts in growth hormone gene therapy to improve growth of midget swine. Hum Gene Ther. 1998 Sep 20;9(14):1995-2003.

53 Hurwitz DR, Kirchgesser M, Merrill W, Galanopoulos T, Mcgrath CA, Emami S, et al. Systemic delivery of human growth hormone or human factor IX in dogs by reintroduced genetically modified autologous bone marrow stromal cells. Hum Gene Ther. 1997 Jan 20;8(2):137-56.

54 Racz GZ, Zheng C, Goldsmith CM, Baum BJ, Cawley NX. Toward gene therapy for growth hormone deficiency via salivary gland expression of growth hormone. Oral Dis. 2015 Mar 1;21(2):149-55.

55 Higuti E, Cecchi Kaadt C, Lima E, Aagaard L, Jensen T, Bartolini P, et al. Early treatment for growth hormone deficiency based on naked DNA administration in dwarf mice allows efficient catch-up growth. Mol Therapy. $2015 ; 23: S 71$.
56 Garrahy A, Moran C, Thompson CJ. Diagnosis and management of central diabetes insipidus in adults. Clin Endocrinol. 2019 Jan 1;90(1):23-30.

57 Qureshi S, Galiveeti S, Bichet DG, Roth J. Diabetes insipidus: celebrating a century of vasopressin therapy. Endocrinology. $2014 \mathrm{Dec}$ 1;155(12):4605-21.

58 Geddes BJ, Harding TC, Lightman SL, Uney JB. Long-term gene therapy in the CNS: reversal of hypothalamic diabetes insipidus in the Brattleboro rat by using an adenovirus expressing arginine vasopressin. Nat Med. 1997;3(12):1402-4.

59 Yoshida M, Iwasaki Y, Asai M, Nigawara T, Oiso Y. Gene therapy for central diabetes insipidus: effective antidiuresis by muscle-targeted gene transfer. Endocrinology. 2004 Jan 1;145(1):261-8.

60 Speised PW, Arlt W, Auchus RJ, Baskin LS, Conway GS, Merke DP, et al. Congenital adrenal hyperplasia due to steroid 21-hydroxylase deficiency: an Endocrine Society* clinical practice guideline. J Clin Endocrinol Metab. 2018 Nov 7;104(1):4043-88.

61 Mnif MF, Kamoun M, Mnif F, Charfi N, Kallel N, Ben Naceur B, et al. Long-term outcome of patients with congenital adrenal hyperplasia due to 21-hydroxylase deficiency. Am J Med Sci. 2012 Nov 1;344(5):363-73.

62 Ali SR, Bryce J, Haghpanahan H, Lewsey JD, Tan LE, Atapattu N, et al. Real-world estimates of adrenal insufficiency-related adverse events in children with congenital adrenal hyperplasia. J Clin Endocrinol Metab. 2021 Jan 1;106(1):e192-203.

63 Tresoldi AS, Sumilo D, Perrins M, Toulis KA, Prete A, Reddy N, et al. Increased infection risk in addison's disease and congenital adrenal hyperplasia: a primary care database cohort study. J Clin Endocrinol Metab. 2020 Feb 1;105(2):418-29.

64 Jenkins-Jones S, Parviainen L, Porter J, Withe M, Whitaker MJ, Holden SE, et al. Poor compliance and increased mortality, depression and healthcare costs in patients with congenital adrenal hyperplasia. Eur J Endocrinol. 2018 Apr;178(4):309-20.

65 Tajima T, Okada T, Ma XM, Ramsey W, Bornstein S, Aguilera G. Restoration of adrenal steroidogenesis by adenovirus-mediated transfer of human cytochromeP450 21-hydroxylase into the adrenal gland of 21-hydroxylase-deficient mice. Gene Ther. 1999; 6(11):1898-903.

66 Perdomini M, Dos Santos C, Goumeaux C, Blouin V, Bougnères P. An AAVrh10-CAGCYP21-HA vector allows persistent correction of 21-hydroxylase deficiency in a Cyp21-/- mouse model. Gene Ther. 2017; 24(5):275-81.

67 Eclov RJ, Lewis TEW, Kapadia M, Scott DW, McCoy DD, Rouse JL, et al. OR25-01 durable CYP21A2 gene therapy in non-human primates for treatment of congenital adrenal hyperplasia. J Endocr Soc. 2020 May 8; 4(Suppl 1):OR25-01.
68 Merke DP, Auchus RJ, Sarafoglou K, Geffner ME, Kim MS, Escandon RD, et al. Design of a phase $1 / 2$ open-label, dose-escalation study of the safety and efficacy of gene therapy in adults with classic congenital adrenal hyperplasia (CAH) due to 21-hydroxylase deficiency through administration of an adenoassociated virus (AAV) serotype 5-based recombinant vector encoding the human CYP21A2 gene. J Endocr Soc. 2021 May 1; 5(Suppl 1):A82.

69 Delmonte OM, Castagnoli R, Calzoni E, Notarangelo LD. Inborn errors of immunity with immune dysregulation: from bench to bedside. Front Pediatr. 2019;7:353.

70 Passerini L, Barzaghi F, Curto R, Sartirana C, Barera G, Tucci F, et al. Treatment with rapamycin can restore regulatory $\mathrm{T}$-cell function in IPEX patients. J Allergy Clin Immunol. 2020 Apr 1;145(4):1262-71.e13.

71 Barzaghi F, Amaya Hernandez LC, Neven B, Ricci S, Kucuk ZY, Bleesing JJ, et al. Longterm follow-up of IPEX syndrome patients after different therapeutic strategies: an international multicenter retrospective study. J Allergy Clin Immunol. 2018 Mar 1;141(3): 1036-49.e5.

72 Sato Y, Passerini L, Piening BD, Uyeda MJ, Goodwin M, Gregori S, et al. Human-engineered treg-like cells suppress FOXP3-deficient $\mathrm{T}$ cells but preserve adaptive immune responses in vivo. Clin Transl Immunology. 2020 Nov 25;9(11):e1214.

73 Goodwin M, Lee E, Lakshmanan U, Shipp S, Froessl L, Barzaghi F, et al. CRISPR-based gene editing enables FOXP3 gene repair in IPEX patient cells. Sci Adv. 2020 May;6(19): eaaz0571.

74 Rees HA, Liu DR. Base editing: precision chemistry on the genome and transcriptome of living cells. Nat Rev Genet. 2018;19(12): 770-88.

75 Anzalone AV, Randolph PB, Davis JR, Sousa AA, Koblan LW, Levy JM, et al. Search-andreplace genome editing without doublestrand breaks or donor DNA. Nature. 2019; 576(7785):149-57.

76 Nakamura M, Gao Y, Dominguez AA, Qi LS. CRISPR technologies for precise epigenome editing. Nat Cell Biol. 2021;23(1):11-22.

77 Wei T, Cheng Q, Min YL, Olson EN, Siegwart DJ. Systemic nanoparticle delivery of CRISPR-Cas9 ribonucleoproteins for effective tissue specific genome editing. Nat Commun. 2020;11(1):3232.

78 Gillmore JD, Gane E, Taubel J, Kao J, Fontana M, Maitland ML, et al. CRISPR-Cas9 in vivo gene editing for transthyretin amyloidosis. N Engl J Med. 2021 Jun 26;385(6):493502.

79 Roncarolo MG, Gregori S, Bacchetta R, Battaglia M, Gagliani N. The biology of T regulatory type 1 cells and their therapeutic application in immune-mediated diseases. Immunity. 2018 Dec 18;49(6):1004-19.
Gene Therapy in Pediatric Endocrine Disease
Horm Res Paediatr 
80 Bluestone JA, Buckner JH, Fitch M, Gitelman SE, Gupta S, Hellerstein MK, et al. Type 1 diabetes immunotherapy using polyclonal regulatory T cells. Sci Transl Med. $2015 \mathrm{Nov}$ 25;7(315):315ra189.

81 Russo F, Citro A, Squeri G, Sanvito F, Monti $\mathrm{P}$, Gregori S, et al. InsB9-23 gene transfer to hepatocyte-based combined therapy abrogates recurrence of type 1 diabetes after islet transplantation. Diabetes. 2021 Jan 1;70(1): $171-81$.
82 Hacein-Bey-Abina S, von Kalle C, Schmidt M, Le Deist F, Wulffraat N, McIntyre E, et al. A serious adverse event after successful gene therapy for X-linked severe combined immunodeficiency. N Engl J Med. 2003 Jan 16; 348(3):255-6.

83 Stein S, Ott MG, Schultze-Strasser S, Jauch A, Burwinkel B, Kinner A, et al. Genomic instability and myelodysplasia with monosomy 7 consequent to EVI1 activation after gene therapy for chronic granulomatous disease. Nat Med. 2010 Feb;16(2):198-204.

84 Servick K. Gene therapy clinical trial halted as cancer risk surfaces [Internet]. 2022. Available from: https://www.science.org/ content/article/gene-therapy-clinical-trialhalted-cancer-risk-surfaces.
85 US Food and Administration. Food and Drug Administration (FDA) cellular, tissue, and gene therapies advisory committee (CTGTAC) meeting \#70 toxicity risks of adenoassociated virus (AAV) vectors for gene therapy (GT) [Internet] September 2-3, 2021. 2021. Available from: https://www.fda.gov/ media/151969/download.

86 Colella P, Ronzitti G, Mingozzi F. Emerging issues in AAV-mediated in vivo gene therapy. Mol Ther Methods Clin Dev. 2017 Dec 1; 8:87-104. 